\title{
Các yếu tố ảnh hưởng đến hành vi công dân tổ chức của nhân viên trong lĩnh vực dịch vụ nhà hàng: Nghiên cứu trường hợp tại Thành phố Hồ Chí Minh
}

\section{Factors affecting organizational citizenship behavior of employees in restaurant service industry: The case of Ho Chi Minh City}

\author{
Nguyễn Hoàng Kim ${ }^{1}$, Nguyễn Lê Hoàng Long ${ }^{1 *}$ \\ ${ }^{1}$ Trường Đại học Kinh tế Thành phố Hồ Chí Minh, Việt Nam \\ *Tác giả liên hệ, Email: longnlh@ueh.edu.vn
}

THÔNG TIN

DOI: $10.46223 / \mathrm{HCMCOUJS}$ econ.vi.16.1.1384.2021

Ngày nhận: 28/05/2020

Ngày nhận lại: 27/10/2020

Duyệt đăng: 29/12/2020

Tù khóa:

cảm nhận sự hỗ trợ của tổ chức, cam kết cảm xúc, dịch vụ nhà hàng, hành vi công dân tổ chức, sự dấn thân vào công việc

Keywords:

affective commitment, job involvement, organizational citizenship behavior, perceived organizational support, restaurant service

\section{TÓM TÁT}

Hành vi công dân tổ chức là khái niệm thể hiện những hành vi tùy ý của cá nhân nhưng mang lại lợi ích cho tổ chức. Trong lĩnh vực dịch vụ nhà hàng, hành vi công dân tổ chức ngày càng được quan tâm nghiên cứu vì ảnh hưởng tích cực của yếu tố này đến sự hài lòng của khách hàng. Tuy nhiên, có rất ít nghiên cứu tìm hiểu cơ chế tác động giữa cảm nhận của nhân viên đối với sự hỗ trợ, quan tâm của tổ chức và hành vi công dân tổ chức. Do đó, nghiên cứu này bổ sung vào các nghiên cứu trước đó qua việc sử dụng mô hình phương trình cấu trúc để kiểm định các giả thuyết có liên quan đến các yếu tố có thể ảnh hưởng đến hành vi công dân tổ chức trong lĩnh vực dịch vụ nhà hàng. Kết quả cho thấy hành vi công dân tổ chức chịu tác động trực tiếp bởi cảm nhận sự hỗ trợ của tổ chức và gián tiếp thông qua sự dấn thân vào công việc và cam kết cảm xúc.

\begin{abstract}
Organizational citizenship behavior (OCB) refers to out-role behaviors but bring benefits to the organization. OCB is being popular researched in restaurant service because of the positive effect on customer satisfaction. However, there are few studies about the mechanism of nexus between perceived organizational support and organizational citizenship behavior. This study aims to contribute to the literature about OCB in the restaurant service industry by adopting Structural Equation Modeling (SEM) for testing hypotheses. The results showed that OCB is directly affected by perceived organizational support and indirectly influenced through job involvement, affective commitment.
\end{abstract}

\section{Mở đầu}

Thực tiễn hoạt động quản trị nguồn nhân lực cho thấy một trong những yếu tố quan trọng làm giảm đi tính hiệu quả của một tổ chức là vấn đề nhân viên chỉ cố gắng hoàn thành vai công việc của mình (Ryan, Schmit, \& Johnson, 1996). Hiện tượng này xuất phát từ việc giữa người 
nhân viên và tổ chức không có nhiều sự tương tác trực tiếp mà chủ yếu hoạt động dựa trên các công cụ đánh giá mang tính định lượng (Antonsen, 2014). Trong lĩnh vực kinh doanh dịch vụ nhà hàng hiếm khi bắt gặp trường hợp người nhân viên làm nhiều hơn vai trò của mình, sẵn sàng cho những công việc ngoài phân công dù chính những công việc đó giúp gia tăng lợi thế cạnh tranh của tổ chức (Karatepe \& Sokmen, 2006).

Những hành vi quan sát được tại các đơn vị kinh doanh nhà hàng trên địa bàn Thành phố Hồ Chí Minh cho thấy hành vi công dân tổ chức không thật sự nổi bật, trong khi một tổ chức muốn phát triển tốt, tạo sự hài lòng cho khách hàng đòi hỏi các cá nhân phải vượt lên trên những công việc hàng ngày mà họ thường làm, sẵn sàng cống hiến nhiều hơn cho tổ chức (Katz, 1964). Hành vi công dân tổ chức là những hành vi cá nhân tùy ý, không trực tiếp, rõ ràng và không được công nhận bởi hệ thống khen thưởng chính thức nhưng chính những hành vi này giúp thúc đẩy hiệu quả của tổ chức (Organ, 1988). Do đó, hành vi công dân tổ chức là một hiện tượng không được thể chế hóa thành quy định mà xuất phát từ sự tự phát của nhân viên.

Lý thuyết sự hỗ trợ của tổ chức (Organizational support theory) do Eisenberger, Huntington, Hutchison, và Sowa (1986) đề xuất phát biểu rằng chính sự hỗ trợ mà tổ chức dành cho nhân viên sẽ khiến họ cảm thấy có nghĩa vụ phải quan tâm đến sự thịnh vượng của tổ chức và từ đó làm bật ra hành vi quan trọng với tổ chức gọi là hành vi công dân. Ngoài ra, sự quan tâm của tổ chức đến nhân viên cũng có thể khiến họ dấn thân hơn vào công việc đang làm, mang lại sự cam kết về mặt cảm xúc để từ đó xuất hiện những hành vi ngoài vai trò (Cho \& Johanson, 2008; Guchait \& Back, 2016).

Một số nghiên cứu gần đây đã tìm hiểu tác động của cảm nhận sự hỗ trợ của tổ chức đến hành vi công dân tổ chức (Afsar \& Badir, 2016; Dai, Hou, Chen, \& Zhuang, 2018; Thompson, Fidell, \& Ullman, 2020), mặc dù vậy các nghiên cứu chỉ tiến hành khảo sát độc lập ảnh hưởng này qua sự dấn thân vào công việc (Kim, 2013) hoặc cam kết cảm xúc (Gupta, Agarwal, \& Khatri, 2016). Nghiên cứu này đóng góp vào khoảng trống ở những nghiên cứu trước đó qua việc đồng thời xem xét vai trò của sự dấn thân vào công việc và cam kết cảm xúc của nhân viên trong mối quan hệ giữa cảm nhận sự hỗ trợ của tổ chức và hành vi công dân tổ chức thuộc lĩnh vực dịch vụ nhà hàng. Ngoài ra, nghiên cứu cũng đưa ra những hàm ý cho nhà quản lý nhằm có thể thúc đẩy hành vi công tổ chức của nhân viên để từ đó gia tăng hiệu quả hoạt động của tổ chức.

\section{Cơ sở lý thuyết và mô hình nghiên cứu}

\subsection{Co' sở lý thuyết}

\subsubsection{Cảm nhận sự hỗ trợ của tổ chức (Perceived organizational support)}

Cảm nhận sự hỗ trợ của tổ chức là khái niệm thuộc về lý thuyết sự hỗ trợ của tổ chức được Eisenberger và cộng sự (1986) định nghĩa là việc "nhân viên phát triển một niềm tin toàn diện liên quan đến mức độ mà tồ chức coi trọng sự đóng góp và quan tâm đến phúc lợi của họ". Cảm nhận sự hỗ trợ của tổ chức được xem là một sự đảm bảo rằng trong những tình huống phức tạp, tổ chức vẫn sẽ hỗ trợ cho nhân viên nếu cần để họ có thể hoàn thành công việc của mình một cách hiệu quả nhất (George, Reed, Ballard, Colin, \& Fielding, 1993).

\subsubsection{Sụ dấn thân vào công việc (Job involvement)}

Khái niệm dấn thân vào công việc được mô tả lần đầu bởi Lodahl và Kejiner (1965). Đối với những cá nhân có sự dấn thân vào công việc ở mức độ cao, thực hiện tốt công việc là điều quan trọng đối với lòng tự trọng của họ (Lodahl \& Kejiner, 1965). Kanungo (1982) sau đó định nghĩa sự dấn thân vào công việc là mức độ tâm lý mà người lao động xác định hoặc cam kết đối với công việc của mình. Tương tự, Joiner và Bakalis (2006) mô tả sự dấn thân vào công việc thể 
hiện sự quan tâm, say mê của nhân viên đối với các mục tiêu, văn hóa và nhiệm vụ của tổ chức.

\subsubsection{Cam kết cảm xúc (Affective commitment)}

Cam kết cảm xúc đề cập đến sự cống hiến, gắn kết tình cảm đối với tổ chức của nhân viên (Allen \& Meyer, 1990). Cohen (2007) cho rằng cam kết cảm xúc là một dạng tình cảm tích cực đối với tổ chức khi người nhân viên mong đợi tổ chức mình thành công và cảm thấy tự hào khi mình là một phần của tổ chức. Một trong những hệ quả tích cực của cam kết cảm xúc là nhân viên sẽ ước muốn mình tiếp tục là thành viên của tổ chức (Rhoades, Eisenberger, \& Armeli, 2001).

\subsubsection{Hành vi công dân tổ chức (Organizational citizenship behavior)}

Hành vi công dân tổ chức được định nghĩa là những hành vi cá nhân ngoài vai trò và không được công nhận rõ ràng trong hệ thống khen thưởng chính thức tại nơi làm việc (Podsakoff \& MacKenzie, 1994). Nhân viên có mức độ hành vi công dân tổ chức cao sẽ sẵn sàng dấn thân nhiều hơn cho tổ chức (Chen, Hui, \& Sego, 1998) và thể hiện những hành vi tùy nguyện vì tổ chức (Organ, Podsakoff, \& MacKenzie, 2006). Vì vậy, hành vi công dân tổ chức là hành vi mà cá nhân lựa chọn thực hiện nhằm mang lại lợi ích cho người khác, vượt ra ngoài vai trò yêu cầu trong tổ chức và cuối cùng chính những hành vi này sẽ mang lại lợi ích cho tổ chức (Deckop, Cirka, \& Andersson, 2003; Peloza \& Hassay, 2006).

\subsection{Các giả thuyết nghiên cúu}

\subsubsection{Cảm nhận sụ hố trợ của tổ chức và hành vi công dân tổ chức}

Trên khía cạnh trao đổi xã hội, cảm nhận sự hỗ trợ của tổ chức sẽ yêu cầu trách nhiệm từ nhân viên nhằm đáp ứng các mục tiêu của tổ chức, và khi nhân viên cảm thấy rằng tổ chức coi trọng các giá trị cá nhân của mình, họ sẽ đáp lại thông qua các hành vi công dân tổ chức (Moorman, Blakely, \& Niehoff, 1998; Piercy, Cravens, \& Lane, 2012; Podsakoff, MacKenzie, Paine, \& Bachrach, 2000; Settoon, Bennett, \& Liden, 1996). Nghiên cứu của Wayne, Shore, Bommer, và Tetrick (2002) đã cho thấy cảm nhận sự hỗ trợ của tổ chức có tác động mạnh đến hành vi công dân tổ chức. Kết quả nghiên cứu của Cardona, Lawrence, và Bentler (2004) cũng xác nhận rằng những cá nhân có mức độ cảm nhận sự hỗ trợ của tổ chức cao sẽ có được mối quan hệ xã hội vững chắc với tổ chức của họ, từ đó giúp gia tăng các hành vi công dân tổ chức. Vì vậy, cảm nhận sự hỗ trợ của tổ chức được giả định rằng có thể thúc đẩy các hành vi công dân tổ chức:

$H_{1}$ : Cảm nhận sụ hỗ trợ của tổ chức có tác động tích cực đến hành vi công dân tổ chức của nhân viên

\subsubsection{Cảm nhận sụ hỗ trợ của tổ chức và cam kết cảm xúc}

Các yếu tố liên quan đến cảm nhận sự hỗ trợ của tổ chức như tăng lương, đề bạt, đào tạo, phát triển được coi là dấu hiệu mà tổ chức thể hiện sự tôn trọng và quan tâm đến nhân viên, giúp nhân viên nâng cao niềm tin của họ với tổ chức (Chen, Aryee, \& Lee, 2005; Cheung, 2000). Nhân viên sẽ hình thành nên thái độ tích cực đối với tổ chức để thể hiện lòng biết ơn và qua đó làm gia tăng mức độ cam kết tình cảm của họ (Rhoades et al., 2001). Kim, Leong, và Lee (2005) cũng ủng hộ quan điểm này khi cho rằng nhân viên có xu hướng thể hiện cảm xúc mạnh mẽ để mong muốn tiếp tục là thành viên của tổ chức khi họ cảm nhận được có sự hỗ trợ từ tổ chức của mình. Do đó, cảm nhận sự hỗ trợ của tổ chức được dự đoán có thể nâng cao cam kết cảm xúc:

$\mathrm{H}_{2}$ : Cảm nhận sự hỗ trợ của tổ chức có tác động tích cực đến cam kết cảm xúc của nhân viên 


\subsubsection{Cảm nhận sụ hỗ trợ của tổ chức và sụ dấn thân vào công việc}

Lý thuyết trao đổi xã hội cho rằng cá nhân sẽ cảm thấy có nghĩa vụ phải hồi đáp theo cách nào đó khi người khác đối xử tốt với họ (Konovsky \& Pugh, 1994). Bằng chứng thực nghiệm cho thấy các nhân viên có mức độ cảm nhận sự hỗ trợ của tổ chức cao sẽ có trải nghiệm công việc thuận lợi hơn và sẽ đầu tư nhiều hơn cho công việc của họ trong tổ chức (Rhoades \& Eisenberger, 2002). Qua đó, cảm nhận sự hỗ trợ của tổ chức được giả thuyết rằng có thể thúc đẩy sự dấn thân vào công việc:

H3: Cảm nhận sự hỗ trọ của tổ chức có tác động tích cực đến sự dấn thân vào công việc của nhân viên

\subsubsection{Sụ dấn thân vào công việc và cam kết cảm xúc}

Blau và Boal (1987) trong nghiên cứu của mình đã phát hiện rằng có sự tương tác giữa sự dấn thân vào công việc và cam kết tổ chức, theo đó những nhân viên vừa dấn thân vào công việc và vừa cam kết với tổ chức thì nhiều khả năng họ sẽ chọn tiếp tục ở lại tổ chức. Ngoài ra, Mathieu và Zajac (1990) khi nghiên cứu các tiền đề và hệ quả của cam kết tổ chức cũng cho thấy mối quan hệ giữa sự dấn thân vào công việc với cam kết cảm xúc sẽ mạnh hơn so với mối quan hệ đó với cam kết kế tục. Do vậy, sự dấn thân vào công việc được dự đoán có thể giúp gia tăng cam kết cảm xúc:

\section{H4: Sự dấn thân vào công việc có tác động tích cực đến cam kết cảm xúc của nhân viên}

\subsubsection{Cam kết cảm xúc và hành vi công dân tổ chức}

Nhân viên có sự gắn bó về mặt tâm lý là một yếu tố dự báo cho các hành vi công dân tổ chức (Morrison, 1994). Cam kết tổ chức đóng vai trò thiết yếu đối với hành vi công dân tổ chức (Meyer, Stanley, Herscovitch, \& Topolnytsky, 2002) và các nghiên cứu trước đây cũng đã cho thấy mức độ quan trọng của cam kết cảm xúc trong việc xác định các hành vi ngoài vai trò (Meyer et al., 2002). Các cá nhân có cam kết cảm xúc mạnh mẽ sẽ thể hiện các hành vi có lợi cho tổ chức và những hành vi này thường không được tổ chức quy định rõ ràng (Meyer \& Herscovitch, 2001). Vì vậy, giả thuyết cuối cùng có thể được mô tả như sau:

\section{H5: Cam kết cảm xúc có tác động tích cực đến hành vi công dân tổ chức của nhân viên}

Từ các lập luận trên, mô hình nghiên cứu sau được đề xuất:

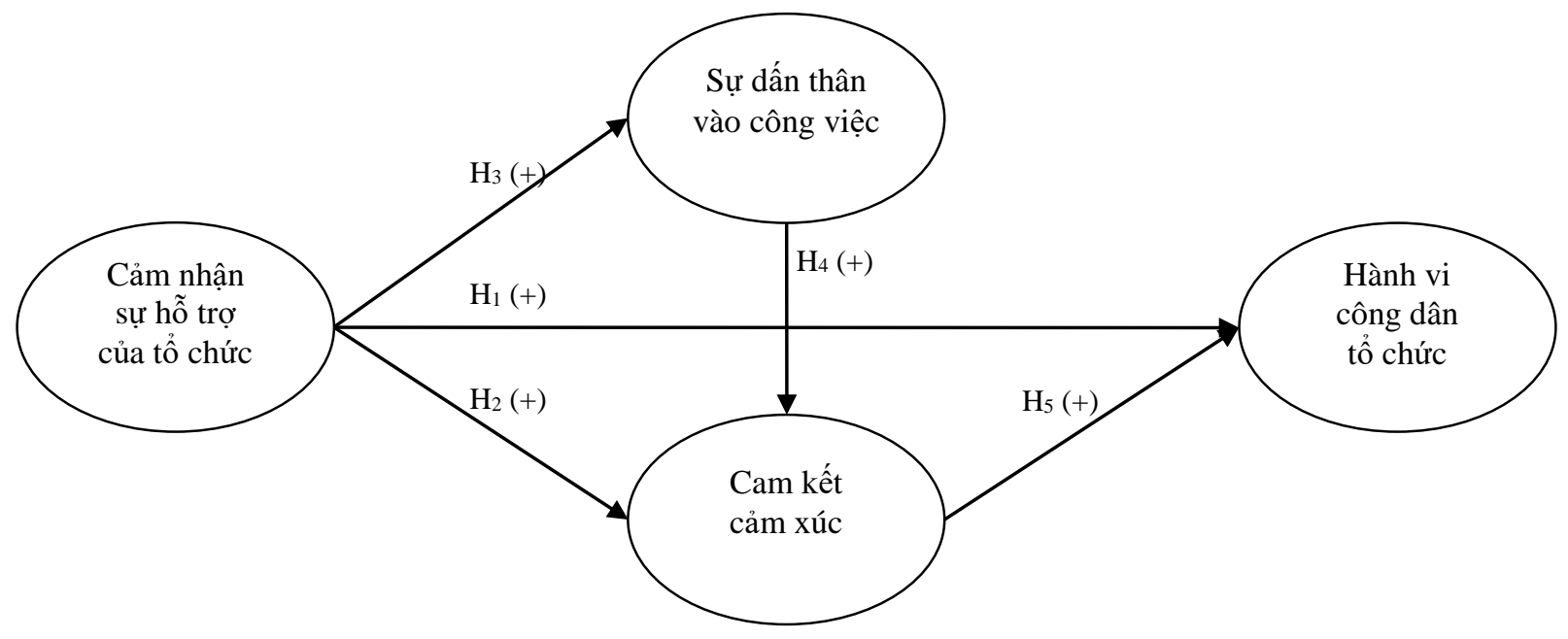

Hình 1. Mô hình nghiên cứu đề xuất 


\section{Thiết kế nghiên cứu}

\subsection{Thang do}

Các thang đo được sử dụng trong bài nghiên cứu được trình bày tại Bảng 1 .

\section{Bảng 1}

Các thang đo trong nghiên cứu

Nội dung thang đo Tác giả

\section{Cảm nhận sự hỗ trợ của tổ chức}

Tổ chức này quan tâm đến tình trạng của tôi.

Tổ chức này có sự cân nhắc mạnh mẽ đến mục tiêu và giá trị của tôi.

Tổ chức này quan tâm đến ý kiến của tôi.

Tổ chức này sẵn sàng giúp đỡ khi tôi thực sự cần.

Sự giúp đỡ luôn hiện diện trong tổ chức khi tôi gặp vấn đề.

Tổ chức này tha thứ những sai sót khách quan của tôi.

\section{Sự dấn thân vào công việc}

Công việc này là một phần quan trọng trong cuộc đời tôi.

Tôi cảm thấy gắn bó về cảm xúc với công việc của mình.

Tôi cảm thấy áy náy nếu rời cơ quan khi công việc chưa hoàn thành.

Tôi cảm thấy thích thú với công việc của mình.

\section{Cam kết cảm xúc}

Tôi sẽ hạnh phúc làm việc tại tổ chức đến khi tôi về hưu.

Rhoades và cộng sự (2001)

Làm việc tại tổ chức có nhiều ý nghĩa đối với cá nhân tôi.

Tôi thật sự cảm thấy vấn đề tổ chức đang đối mặt cũng là vấn đề của tôi.

Tôi cảm nhận có sự gắn kết cá nhân đối với tổ chức của mình.

Tôi tự hào nói cho người khác biết về tổ chức của mình.

Tôi có cảm xúc mạnh mẽ rằng tôi thuộc về tổ chức của mình.

\section{Hành vi công dân tổ chức}

Tôi điều chỉnh lịch làm việc của mình để giúp đỡ các đồng nghiệp khác.

Tôi cố gắng giúp đỡ người khác để họ có thể hòa nhập vào tổ chức.

Singh và Gupta

Eisenberger,

Cummings,

Armeli, và

Lynch (1997)

Tôi tích cực theo dõi sự phát triển của tổ chức.

Ritz, Giauque,

Varone, và

Anderfuhren-

Biget (2014)

Tôi tham gia vào các công việc không được yêu cầu nhưng có thể giúp ích cho hình ảnh của tổ chức.

Tôi đưa ra những gợi ý sáng tạo nhằm cải thiện chức năng của tổ chức.

Nguồn: Nhóm tác giả tổng hợp

\subsection{Dũ liệu nghiên cúu}

Mẫu nghiên cứu được thu thập bằng phương pháp thuận tiện, phi xác suất. Các tác giả sẽ xin phép người quản lý nhà hàng để tiến hành khảo sát và những nhân viên đang làm việc tại các nhà hàng đồng ý tham gia nghiên cứu sẽ được phát bảng câu hỏi giấy. Dữ liệu được phân tích bằng 
phần mềm SPSS ver.26 và AMOS ver.20. Với tổng số 21 biến quan sát, cỡ mẫu tối thiểu cần gấp 5 lần số biến (Bollen, 1989) là 105 quan sát. Người nghiên cứu khảo sát tổng cộng 250 nhân viên, 250 phiếu được thu về, 48 phiếu bị loại do không đạt yêu cầu và cuối cùng 202 phiếu được sử dụng để tiến hành phân tích.

\subsection{Phương pháp phân tích dũ liệu}

Các thang đo được đánh giá độ tin cậy bằng hệ số Cronbach’ Alpha, thang đo đạt yêu cầu khi có hệ số lớn hơn 0,6 và các biến quan sát có tương quan biến - tổng lớn hơn 0,3 (Nunnally \& Bernstein, 1994). Phân tích nhân tố khám phá EFA được thực hiện bằng phương pháp trích Principal Axis Factoring với phép xoay Promax, điểm dừng có hệ số Eigenvalue lớn hơn 1 và tổng phương sai trích lớn hơn 50\% (Anderson \& Gerbing, 1988), biến quan sát sẽ được giữ lại nếu hệ số tải nhân tố lớn hơn 0,5 (Hair, Anderson, Tatham, \& Black, 1998 ) và chênh lệch giữa hệ số tải nhân tố lớn nhất và hệ số tải nhân tố bất kỳ lớn hơn 0,3 . Phân tích nhân tố khẳng định CFA được sử dụng để đánh giá mức độ phù hợp của mô hình với dữ liệu và các giá trị khác. Theo Anderson và Gerbing (1988), nhân tố đạt được giá trị hội tụ nếu các trọng số chuẩn hóa lớn hơn 0,5 và có ý nghĩa thống kê, ngoài ra hai nhân tố sẽ đạt giá trị phân biệt nếu hệ số tương quan khác 1 và có ý nghĩa thống kê (Steenkamp \& Van Trijp, 1991). Bên cạnh đó, giá trị về độ tin cậy tổng hợp CR yêu cầu lớn hơn 0,7 (Bagozzi \& Yi, 1988) và phương sai trích AVE lớn hơn 0,5 (Fornell \& Larcker, 1981). Mô hình được xem là tương thích với dữ liệu khi có các chỉ số: $\chi^{2} / \mathrm{df}$ (Chi-square/Degrees of freedom) nhỏ hơn 2 (Carmines \& McIver, 1981), TLI (Tucker Lewis Index) và CFI (Comparative Fit Index) lớn hơn 0,9 (Tabachnick, Fidell, \& Ullman, 2007), RMSEA (Root Mean Square Error of Approximation) nhỏ hơn 0,08 (Hu \& Bentler, 1999). Để kiểm định các giả thuyết nghiên cứu, mô hình phương trình cấu trúc SEM được sử dụng với các yêu cầu về mức độ phù hợp của mô hình tuân thủ theo các tiêu chuẩn nêu trên.

\section{Kết quả nghiên cứu và thảo luận}

\subsection{Mô tả mẫu nghiên cứu}

Nghiên cứu tiến hành lấy mẫu trên đối tượng là nhân viên phục vụ đang làm việc tại các nhà hàng trên địa bàn Thành phố Hồ Chí Minh, thời gian lấy mẫu trong tháng 03/2020. Các đối tượng được chọn một cách ngẫu nhiên và được hỏi ý kiến đồng ý trước khi chấp nhận tham gia. Việc tạo được sự đồng thuận cũng như xác định đúng đối tượng khảo sát sẽ phản ảnh chính xác độ phù hợp và tạo điều kiện thuận lợi để mở rộng các hướng nghiên cứu về sau (Neuman, 2007).

Trong số các bảng trả lời hợp lệ, phần lớn đội ngũ nhân viên phục vụ có thâm niên công tác từ 1-3 năm (62\%), một số có thời gian trên 5 năm làm nhân viên phục vụ tại nhà hàng $(12 \%)$ và phần còn lại $(26 \%)$ có thời gian công tác dưới một năm. Nhóm nhân viên công tác dưới một năm phần đông là sinh viên, thực hiện công việc toàn thời gian $(58 \%)$ hoặc bán thời gian $(42 \%)$. Tỷ lệ nhân viên là nữ trong số các đối tượng khảo sát có phần vượt trội hơn nam (57\% so với $43 \%)$.

\subsection{Kết quả kiểm định giá trị, độ tin cậy và mức độ phù hợp của mô hình}

Bốn thang đo các khái niệm nghiên cứu qua kiểm định độ tin cậy bằng hệ số Cronbach' Alpha có hệ số biến thiên từ 0,761 (thang đo sự dấn thân vào công việc) đến 0,92 (thang đo cảm nhận sự hỗ trợ của tổ chức) đều cao hơn mức 0,6 và tương quan biến - tổng đều lớn hơn 0,3 (nhỏ nhất là 0,616 ) nên các thang đo đạt yêu cầu về độ tin cậy và không có biến quan sát nào bị loại. Kết quả EFA lần cuối có hệ số KMO bằng $0,909(\mathrm{Sig} .=0,000)$, tại điểm dừng có Eigenvalue bằng 1,395 và tổng phương sai trích bằng $61,547 \%$ rút trích được bốn nhân tố đại diện cho bốn khái niệm nghiên cứu. Các biến quan sát không bị xáo trộn và nhóm lại theo từng nhân tố như thang đo lý thuyết, hệ số tải nhân tố biến thiên từ 0,568 đến 0,884 . 


\section{Bảng 2}

Kết quả kiểm định giá trị, độ tin cậy

\begin{tabular}{lcccc}
\hline \multicolumn{1}{c}{ Nhân tố } & $\begin{array}{c}\text { Số biến } \\
\text { quan sát }\end{array}$ & $\begin{array}{c}\text { Cronbach’ } \\
\text { Alpha }\end{array}$ & CR & AVE \\
\hline Cảm nhận sự hỗ trợ của tổ chức & 6 & 0,923 & 0,924 & 0,672 \\
Sự dấn thân vào công việc & 4 & 0,833 & 0,837 & 0,563 \\
Cam kết cảm xúc & 6 & 0,872 & 0,874 & 0,539 \\
Hành vi công dân tổ chức & 5 & 0,880 & 0,887 & 0,614 \\
\hline
\end{tabular}

Nguồn: Nhóm tác giả phân tích.

Phân tích nhân tố khẳng định CFA mô hình tới hạn có các chỉ số: $\chi^{2} / \mathrm{df}=299,419 / 419=$ $1,663(\mathrm{p}=0,000), \mathrm{TLI}=0,947, \mathrm{CFI}=0,954$ và $\mathrm{RMSEA}=0,057$ cho thấy mô hình nghiên cứu đề xuất phù hợp với dữ liệu. Hệ số tải của các biến quan sát biến thiên từ 0,65 đến 0,91 đều lớn hơn 0,5 và có ý nghĩa thống kê $(\mathrm{p}=0,000)$, do đó bốn nhân tố đạt được giá trị hội tụ và không có biến quan sát nào bị loại. Hệ số tương quan của từng cặp nhân tố khác biệt so với $1(p=0,000)$ (Bảng 3) nên bốn nhân tố đạt được giá trị phân biệt. Ngoài ra, kết quả cũng cho thấy các giá trị độ tin cậy tổng hợp lớn hơn 0,7 và phương sai trích lớn hơn 0,5 đều đạt yêu cầu (Bảng 2 ).

\section{Bảng 3}

Kết quả kiểm định giá trị phân biệt

\begin{tabular}{lcccccc}
\hline & \multirow{2}{*}{ Mối quan hệ } & $\begin{array}{c}\text { Hệ số } \\
\text { tương quan (r) }\end{array}$ & $\begin{array}{c}\text { Sai lẹ̣ch } \\
\text { chuẩn }\end{array}$ & $\begin{array}{c}\text { Giá trị } \\
\text { tới hạn }\end{array}$ & p \\
\hline Cảm nhận & $<-->$ & Hành vi & 0,392 & 0,033 & 4,515 & 0,000 \\
Cảm nhận & $<-->$ & Cam kết & 0,595 & 0,040 & 5,856 & 0,000 \\
Cảm nhận & $<->$ & Dấn thân & 0,565 & 0,036 & 5,492 & 0,000 \\
Dấn thân & $<-->$ & Cam kết & 0,646 & 0,031 & 6,051 & 0,000 \\
Dấn thân & $<-->$ & Hành vi & 0,469 & 0,026 & 5,007 & 0,000 \\
Cam kết & $<-->$ & Hành vi & 0,377 & 0,027 & 4,369 & 0,000 \\
\hline
\end{tabular}

Nguồn: Nhóm tác giả phân tích

\subsection{Kiểm định giả thuyết nghiên cưu}

Kết quả phân tích mô hình phương trình cấu trúc SEM có các chỉ số: $\chi^{2} / \mathrm{df}=309,283 / 181$ $=1,709(\mathrm{p}=0,000), \mathrm{TLI}=0,943, \mathrm{CFI}=0,951$ và $\mathrm{RMSEA}=0,059$ cho thấy mô hình nghiên cứu phù hợp với dữ liệu. Kết quả kiểm định các mối quan hệ được trình bày ở Bảng 4 . 


\section{Bảng 4}

Kết quả kiểm định các mối quan hệ

\begin{tabular}{llllcccc}
\hline \multicolumn{2}{c}{ Giả thuyết } & \multicolumn{2}{c}{ Mối quan hệ } & $\begin{array}{c}\text { Ước } \\
\text { lượng }\end{array}$ & $\begin{array}{c}\text { Sai lệch } \\
\text { chuẩn }\end{array}$ & $\begin{array}{c}\text { Giá trị } \\
\text { tới hạn }\end{array}$ & p \\
\hline $\mathrm{H}_{1}$ & Cảm nhận & $-->$ & Hành vi & 0,251 & 0,072 & 2,737 & 0,006 \\
$\mathrm{H}_{2}$ & Cảm nhận & $-->$ & Cam kết & 0,331 & 0,067 & 4,040 & 0,000 \\
$\mathrm{H}_{3}$ & Cảm nhận & $-->$ & Dấn thân & 0,567 & 0,064 & 6,616 & 0,000 \\
$\mathrm{H}_{4}$ & Dấn thân & $-->$ & Cam kết & 0,466 & 0,098 & 5,255 & 0,000 \\
$\mathrm{H}_{5}$ & Cam kết & $-->$ & Hành vi & 0,245 & 0,090 & 2,642 & 0,009 \\
\hline
\end{tabular}

Nguồn: Nhóm tác giả phân tích

Năm giả thuyết được đặt ra ban đầu được kiểm định gồm: giả thuyết $\mathrm{H}_{1}$ phát biểu rằng cảm nhận sự hỗ trợ của tổ chức có tác động tích cực đến hành vi công dân tổ chức $(\beta=0,251 ; \mathrm{p}<0,01)$, giả thuyết $\mathrm{H}_{2}$ đề xuất cảm nhận sự hỗ trợ của tổ chức có tác động tích cực đến cam kết cảm xúc ( $\beta$ $=0,331 ; \mathrm{p}<0,000)$, tương tự, giả thuyết $\mathrm{H}_{3}$ nhận định cảm nhận sự hỗ trợ của tổ chức có tác động tích cực đến sự dấn thân vào công việc $(\beta=0,567 ; \mathrm{p}<0,000)$, giả thuyết $\mathrm{H}_{4}$ nhìn nhận sự dấn thân vào công việc có tác động tích cực đến cam kết cảm xúc $(\beta=0,466 ; p<0,000)$, và cuối cùng giả thuyết $\mathrm{H}_{5}$ cho rằng cam kết cảm xúc có tác động tích cực đến hành vi công dân tổ chức $(\beta=0,245$; $\mathrm{p}<0,01)$, kết quả cho thấy các giả thuyết trên đều được chấp nhận.

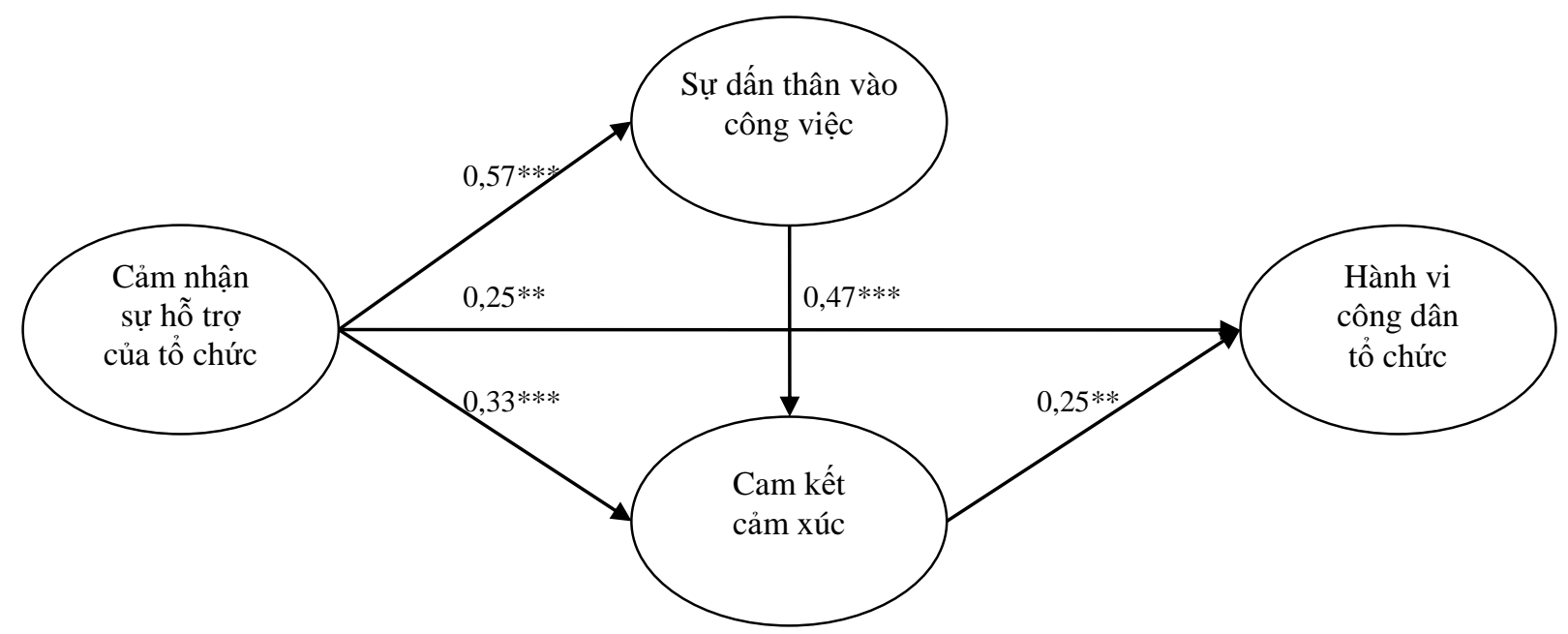

Hình 2. Mô hình nghiên cứu sau cùng

Ghi chú: *** $\mathrm{p}<0,001 \quad * * \mathrm{p}<0,01$

\section{Kết luận và khuyến nghị}

\subsection{Kết luận}

Nghiên cứu cho thấy cảm nhận sự hỗ trợ của tổ chức có tác động tích cực đến hành vi công dân tổ chức theo cách trực tiếp hoặc tác động thông qua cam kết cảm xúc và sự dấn thân vào công việc, đặc biệt trong bối cảnh nghiên cứu ở Việt Nam và đặc thù trong ngành kinh doanh nhà hàng. Nghiên cứu khảo sát trên đối tượng là nhân viên trực tiếp phục vụ khách hàng làm việc theo chế độ toàn thời gian và bán thời gian cho thấy chính sự quan tâm, hỗ trợ trong nhà hàng có thể khiến đội ngũ nhân viên tuyến đầu thể hiện được những hành vi ngoài vai trò trong công việc. Hành vi 
này có thể diễn ra một cách trực tiếp bởi cảm nhận của nhân viên về các chính sách hỗ trợ của tổ chức hoặc thông qua các trải nghiệm về cam kết, hay là một chuỗi quá trình từ việc dấn thân vào công việc đang làm cho đến hình thành nên sự cam kết và từ đó thể hiện ra các hành vi ngoài vai trò.

Nhân viên bán thời gian được coi là nguồn lao động cần thiết và linh hoạt trong lĩnh vực phục vụ ăn uống (Inman \& Enz, 1995), đặc biệt là các nhà hàng vì đây là khu vực sử dụng rất nhiều lao động do nhu cầu dịch vụ luôn biến động. (Enz \& Inman, 1992). Những nhân viên bán thời gian được coi là một lợi thế trong ngành này vì lịch làm việc linh hoạt cũng như việc tiết kiệm tiền lương và phúc lợi chi trả, mặc dù vậy những nhân viên này lại thường được yêu cầu thực hiện các nhiệm vụ công việc và có trách nhiệm giống như nhân viên toàn thời gian (Stamper \& Van Dyne, 2003), tuy nhiên trong quá khứ họ bị xem là không đạt tiêu chuẩn so với lao động toàn thời gian với tỷ lệ nghỉ việc cao hơn, không được đánh giá cao hoặc không được đào tạo đầy đủ (Inman \& Enz, 1995; Tilly, 1992).

Ngày nay, nhân viên bán thời gian được coi là yếu tố có thể giúp đạt được lợi thế cạnh tranh khi các đơn vị dịch vụ nhà hàng cố gắng tạo ra những cách thức mới nhằm tối đa hóa nỗ lực làm việc của nhằm nâng cao chất lượng dịch vụ và giữ chân khách hàng (Stamper \& Van Dyne, 2003). Khi tổ chức đối xử một cách trân trọng và quan tâm đến phúc lợi của nhân viên, người nhân viên sẽ có xu hướng hình thành tâm lý gắn bó và sự cam kết để thúc đẩy họ vượt qua những gì họ phải làm (Van Dyne \& Ang, 1998). Với những thách thức ngày càng tăng trong ngành công nghiệp dịch vụ (Bock, Mangus, \& Folse, 2016), nghiên cứu này cho thấy sự hỗ trợ của tổ chức đã thể hiện ảnh hưởng đến những kết quả liên quan đến công việc, do đó nêu bật tầm quan trọng của việc kiểm định các mối quan hệ thuộc cấp độ cá nhân như sự dấn thân vào công việc, cam kết cảm xúc để có thể hiểu được những tác động này đến hành vi công dân tổ chức của nhân viên trong lĩnh vực dịch vụ nhà hàng.

\subsection{Khuyến nghị}

Kết quả nghiên cứu đã cho thấy những yếu tố xuất phát từ sự quan tâm của tổ chức có thể làm gia tăng những hành vi ngoài vai trò của nhân viên, qua đó góp phần gia tăng chất lượng phục vụ và hiệu quả công việc. Vì vậy, một số khuyến nghị cần thiết cho nhà quản lý được đề xuất như sau:

- Đối với trương ca: cần thiết xây dựng một hệ thống chính sách mang tính trao quyền cho trưởng ca. Người nắm giữ vị trí này cần được huấn luyện về văn hóa chia sẻ trong tổ chức, góp phần lan tỏa sự hỗ trợ đến nhân viên cấp dưới để có thể làm họ thể hiện những hành vi ngoài vai trò;

- Đối với giám sát: vị trí giám giám sát cần là cầu nối giữa trưởng ca và nhân viên phục vụ, làm giảm đi khoảng cách quyền lực giữa quản lý và nhân viên. Người giám sát là đối tượng trực tiếp có thể ảnh hưởng đến cảm nhận của nhân viên cấp dưới đối với tổ chức, do đó cần xây dựng một bộ quy tắc ứng xử chung trong toàn tổ chức với trọng tâm lấy văn hóa hỗ trợ làm tiêu chí thiết lập chính;

- Đối với nhân viên phục vụ: các nhà quản lý trong khu vực nhà hàng cần cải thiện chính sách đối với những nhân viên làm ca kíp, tuân thủ những phúc lợi đã đề ra như chính sách du lịch, nghỉ mát hàng năm. Ngoài ra, đơn vị có thể thiết lập một quỹ với cơ chế minh bạch để có sử dụng vào các hoạt động hỗ trợ nhân viên, giúp họ an tâm làm việc, qua đó có thể làm thay đổi tích cực về mặt cảm xúc và làm xuất hiện hành công dân trong tổ chức. 


\section{Hạn chế và hướng nghiên cứu tiếp theo}

Bên cạnh những kết quả đạt được thì nghiên cứu này vẫn còn một số hạn chế cần thiết phải có những nghiên cứu tiếp theo để có thể hoàn thiện cơ sở lý luận về hành vi công dân tổ chức đối với lĩnh vực kinh doanh dịch vụ nhà hàng, cụ thể như sau:

Thứ nhất, nghiên cứu thu nhập dữ liệu cắt ngang trong tháng 03/2020, đây là thời điểm mà kinh tế - xã hội Việt Nam chịu nhiều ảnh hưởng do dịch bệnh, hoạt động kinh doanh lĩnh vực nhà hàng bị sụt giảm, do đó đội ngũ nhân viên hiện đang làm việc có thể có xu hướng thể hiện nhiều hơn những hành vi ngoài vai trò trong khoảng thời gian này để đảm bảo vị trí công việc. Các nghiên cứu về sau cần có khoảng thời gian thu thập dữ liệu dài hơn để tổng quát được kết quả nghiên cứu.

Thứ hai, hành vi công dân tổ chức ngoài chịu tác động từ các yếu tố như cảm nhận của nhân viên về sự hỗ trợ của tổ chức hay cam kết cảm xúc còn có thể xuất phát từ nhiều yếu tố khác cần thiết phải làm rõ thêm. Ngoài ra, một số nghiên cứu đã khảo sát sự khác biệt về cam kết tổ chức và hành vi công dân tổ chức giữa nhân viên toàn thời gian và bán thời gian (Chang \& Chelladurai, 2003; Feather \& Rauter, 2004; Stamper \& Van Dyne, 2001, 2003), tuy nhiên những kết quả này chưa có sự nhất quán. Do đó các nhà nghiên cứu có thể làm rõ hơn vai trò của các yếu tố này trong các nghiên cứu tiếp theo.

Thứ ba, nghiên cứu được tiến hành tại khu vực Thành phố Hồ Chí Minh với đặc thù là mật độ cư dân đông và điều kiện kinh tế khác biệt so với nhiều tỉnh, thành khác. Các nghiên cứu tiếp theo cần đa dạng hóa những bối cảnh nghiên cứu khác nhau.

\section{Tài liệu tham khảo}

Afsar, B., \& Badir, Y. F. (2016). Person-organization fit, perceived organizational support, and organizational citizenship behavior: The role of job embeddedness. Journal of Human Resources in Hospitality \& Tourism, 15(3), 252-278.

Allen, N. J., \& Meyer, J. P. (1990). The measurement and antecedents of affective, continuance and normative commitment to the organization. Journal of Occupational Psychology, 63(1), 1-18.

Anderson, J. C., \& Gerbing, D. W. (1988). Structural equation modeling in practice: A review and recommended two-step approach. Psychological Bulletin, 103(3), 411-423.

Antonsen, Y. (2014). The downside of the balanced scorecard: A case study from Norway. Scandinavian Journal of Management, 30(1), 40-50.

Bagozzi, R. P., \& Yi, Y. (1988). On the evaluation of structural equation models. Journal of the Academy of Marketing Science, 16(1), 74-94.

Blau, G., \& Boal, K. B. (1987). Conceptualizing how job involvement and organizational commitment affect turnover and absenteeism. Academy of Managernent, 12(2), 288-300.

Bock, D. E., Mangus, S. M., \& Folse, J. A. G. (2016). The road to customer loyalty paved with service customization. Journal of Business Research, 69(10), 3923-3932.

Bollen, K. A. (1989). A new incremental fit index for general structural equation models. Sociological Methods \& Research, 17(3), 303-316. 
Cardona, P., Lawrence, B. S., \& Bentler, P. M. (2004). The influence of social and work exchange relationships on organizational citizenship behavior. Group \& Organization Management, 29(2), 219-247.

Carmines, E. G., \& McIver, J. P. (1981). Analyzing models with unobserved variables. In G. W. Bohrnstedt \& E. F. Borgatta (Eds.), Social measurement: Current issues. Beverly Hills, CA: Sage Publications.

Chang, K., \& Chelladurai, P. (2003). Comparison of part-time workers and full-time workers: Commitment and citizenship behaviors in Korean sport organizations. Journal of Sport Management, 17(4), 394-416.

Chen, X. P., Hui, C., \& Sego, D. J. (1998). The role of organizational citizenship behavior in turnover: Conceptualization and preliminary tests of key hypotheses. Journal of Applied Psychology, 83(6), 922-931.

Chen, Z. X., Aryee, S., \& Lee, C. (2005). Test of a mediation model of perceived organizational support. Journal of Vocational Behavior, 66(3), 457-470.

Cheung, C. K. (2000). Commitment to the organization in exchange for support from the organization. Social Behavior and Personality: An International Journal, 28(2), 125-140.

Cho, S., \& Johanson, M. M. (2008). Organizational citizenship behavior and employee performance: A moderating effect of work status in restaurant employees. Journal of Hospitality \& Tourism Research, 32(3), 307-326.

Cohen, A. (2007). Commitment before and after: An evaluation and reconceptualization of organizational commitment. Human Resource Management Review, 17(3), 336-354.

Cropanzano, R., Howes, J. C., Grandey, A. A., \& Toth, A. P. (1997). The relationship of organizational politics and support to work behaviors, attitudes and stress. Journal of Organizational Behavior, 18(2), 159-180.

Dai, Y. D., Hou, Y. H., Chen, K. Y., \& Zhuang, W. L. (2018). To help or not to help: Antecedents of hotel employees' organizational citizenship behavior. International Journal of Contemporary Hospitality Management, 30(3), 1293-1313.

Deckop, J. R., Cirka, C. C., \& Andersson, L. M. (2003). Doing unto others: The reciprocity of helping behavior in organizations. Journal of Business Ethics, 47(2), 101-113.

Eisenberger, R., Cummings, J., Armeli, S., \& Lynch, P. (1997). Perceived organizational support, discretionary treatment, and job satisfaction. Journal of Applied Psychology, 82(5), 812-820.

Eisenberger, R., Huntington, R., Hutchison, S., \& Sowa, D. (1986). Perceived organizational support. Journal of Applied Psychology, 71(3), 500-507.

Eisenberger, R., Stinglhamber, F., Vandenberghe, C., Sucharski, I. L., \& Rhoades, L. (2002). Perceived supervisor support: Contributions to perceived organizational support and employee retention. Journal of Applied Psychology, 87(3), 565-573.

Enz, C., \& Inman, C. (1992). A comparison of attitudes and work practices of part-time and fulltime workers in the food service industry. In Annual CHRIE conference proceedings (pp. 19-21). Washington, DC: CHRIE.

Feather, N. T., \& Rauter, K. A. (2004). Organizational citizenship behaviours in relation to job status, job insecurity, organizational commitment and identification, job satisfaction and work values. Journal of Occupational and Organizational Psychology, 77(1), 81-94. 
Fornell, C., \& Larcker, D. F. (1981). Evaluating structural equation models with unobservable variables and measurement error. Journal of Marketing Research, 18(1), 39-50.

George, J. M., Reed, T. F., Ballard, K. A., Colin, J., \& Fielding, J. (1993). Contact with AIDS patients as a source of work-related distress: Effects of organizational and social support. Academy of Management Journal, 36(1), 157-171.

Guchait, P., \& Back, K. J. (2016). Three country study: Impact of support on employee attitudes. The Service Industries Journal, 36(7/8), 299-318.

Gupta, V., Agarwal, U. A., \& Khatri, N. (2016). The relationships between perceived organizational support, affective commitment, psychological contract breach, organizational citizenship behaviour and work engagement. Journal of Advanced Nursing, 72(11), 2806-2817.

Hair, J., Anderson, R., Tatham, R., \& Black, W. (1998). Multivariate data analysis (5th ed.). Upper Saddle River, NJ: Prentice Hall.

Hu, L. T., \& Bentler, P. M. (1999). Cutoff criteria for fit indexes in covariance structure analysis: Conventional criteria versus new alternatives. Structural Equation Modeling: A Multidisciplinary Journal, 6(1), 1-55.

Inman, C., \& Enz, C. (1995). Shattering the myths of the part-time worker. Cornell Hotel and Restaurant Administration Quarterly, 36(5), 70-73.

Joiner, T. A., \& Bakalis, S. (2006). The antecedents of organizational commitment: The case of Australian casual academics. International Journal of Educational Management, 20, 439-452.

Kanungo, R. N. (1982). Measurement of job and work involvement. Journal of Applied Psychology, 67(3), 341-349.

Karatepe, O. M., \& Sokmen, A. (2006). The effects of work role and family role variables on psychological and behavioral outcomes of frontline employees. Tourism Management, 27(2), 255-268.

Katz, D. (1964). The motivational basis of organizational behavior. Behavioral Science, 9(2), 131-146.

Kim, M. S. (2013). Effects of hospital nurses' perceived organizational support on job involvement and organizational citizenship behavior. Journal of Korean Academy of Nursing Administration, 19(4), 480-490.

Kim, W. G., Leong, J. K., \& Lee, Y. K. (2005). Effect of service orientation on job satisfaction, organizational commitment, and intention of leaving in a casual dining chain restaurant. International Journal of Hospitality Management, 24(2), 171-193.

Konovsky, M. A., \& Pugh, S. D. (1994). Citizenship behavior and social exchange. Academy of Management Journal, 37(3), 656-669.

Lodahl, T. M., \& Kejnar, M. (1965). The definition and measurement of job involvement. Journal of Applied Psychology, 49(1), 24-33.

Mathieu, J. E., \& Zajac, D. M. (1990). A review and meta-analysis of the antecedents, correlates, and consequences of organizational commitment. Psychological Bulletin, 108(2), 171-194. 
Meyer, J. P., \& Herscovitch, L. (2001). Commitment in the workplace: Toward a general model. Human Resource Management Review, 11(3), 299-326.

Meyer, J. P., Stanley, D. J., Herscovitch, L., \& Topolnytsky, L. (2002). Affective, continuance, and normative commitment to the organization: A meta-analysis of antecedents, correlates, and consequences. Journal of Vocational Behavior, 61(1), 20-52.

Moorman, R. H., Blakely, G. L., \& Niehoff, B. P. (1998). Does perceived organizational support mediate the relationship between procedural justice and organizational citizenship behavior? Academy of Management Journal, 41(3), 351-357.

Morrison, E. W. (1994). Role definitions and organizational citizenship behavior: The importance of the employee's perspective. Academy of Management Journal, 37(6), 1543-1567.

Neuman, L. W. (2007). Social research methods. London, UK: Pearson Education India.

Nunnally, J. C., \& Bernstein, I. H. (1994). Psychometric theory. New York, NY: McGraw-Hill.

Organ, D. W. (1988). Issues in organization and management series. Organizational citizenship behavior: The good soldier syndrome. Lexington, MA: Lexington Books/D. C. Heath and Company.

Organ, D., Podsakoff, P., \& MacKenzie, S. (2006). Organizational citizenship behavior: Its nature, antecedents, and consequences. Thousand Oaks, CA: Sage Publications.

Peloza, J., \& Hassay, D. N. (2006). Intra-organizational volunteerism: Good soldiers, good deeds and good politics. Journal of Business Ethics, 64(4), 357-379.

Piercy, N. F., Cravens, D. W., \& Lane, N. (2012). Sales manager behavior-based control and salesperson performance: The effects of manager control competencies and organizational citizenship behavior. Journal of Marketing Theory and Practice, 20(1), 7-22.

Podsakoff, P. M., \& MacKenzie, S. B. (1994). An examination of the psychometric properties and nomological validity of some revised and reduced substitutes for leadership scales. Journal of Applied Psychology, 79(5), 702-713.

Podsakoff, P. M., MacKenzie, S. B., Paine, J. B., \& Bachrach, D. G. (2000). Organizational citizenship behaviors: A critical review of the theoretical and empirical literature and suggestions for future research. Journal of Management, 26(3), 513-563.

Rhoades, L., \& Eisenberger, R. (2002). Perceived organizational support: A review of the literature. Journal of Applied Psychology, 87(4), 698-714.

Rhoades, L., Eisenberger, R., \& Armeli, S. (2001). Affective commitment to the organization: The contribution of perceived organizational support. Journal of Applied Psychology, 86(5), 825836.

Ritz, A., Giauque, D., Varone, F., \& Anderfuhren-Biget, S. (2014). From leadership to citizenship behavior in public organizations: When values matter. Review of Public Personnel Administration, 34(2), 128-152.

Ryan, A. M., Schmit, M. J., \& Johnson, R. (1996). Attitudes and effectiveness: Examining relations at an organizational level. Personnel Psychology, 49(4), 853-882.

Settoon, R. P., Bennett, N., \& Liden, R. C. (1996). Social exchange in organizations: Perceived organizational support, leader-member exchange, and employee reciprocity. Journal of Applied Psychology, 81(3), 219-227. 
Singh, A., \& Gupta, B. (2015). Job involvement, organizational commitment, professional commitment, and team commitment. Benchmarking: An International Journal, 22(6), 1192-1211.

Stamper, C. L., \& Dyne, L. V. (2001). Work status and organizational citizenship behavior: A field study of restaurant employees. Journal of Organizational Behavior: The International Journal of Industrial, Occupational and Organizational Psychology and Behavior, 22(5), 517-536.

Stamper, C. L., \& Van Dyne, L. (2003). Organizational citizenship: A comparison between parttime and full-time service employees. Cornell Hotel and Restaurant Administration Quarterly, 44(1), 33-42.

Steenkamp, J. B. E., \& Van Trijp, H. C. (1991). The use of LISREL in validating marketing constructs. International Journal of Research in marketing, 8(4), 283-299.

Tabachnick, B. G., Fidell, L. S., \& Ullman, J. B. (2007). Using multivariate statistics. Boston, MA: Pearson.

Thompson, P. S., Bergeron, D. M., \& Bolino, M. C. (2020). No obligation? How gender influences the relationship between perceived organizational support and organizational citizenship behavior. Journal of Applied Psychology, 105(11), 1338-1350.

Tilly, C. (1992). Dualism in part-time employment. Industrial Relations: A Journal of Economy and Society, 31(2), 330-347.

Van Dyne, L., \& Ang, S. (1998). Organizational citizenship behavior of contingent workers in Singapore. Academy of Management Journal, 41(6), 692-703.

Wayne, S. J., Shore, L. M., Bommer, W. H., \& Tetrick, L. E. (2002). The role of fair treatment and rewards in perceptions of organizational support and leader-member exchange. Journal of Applied Psychology, 87(3), 590-598. 